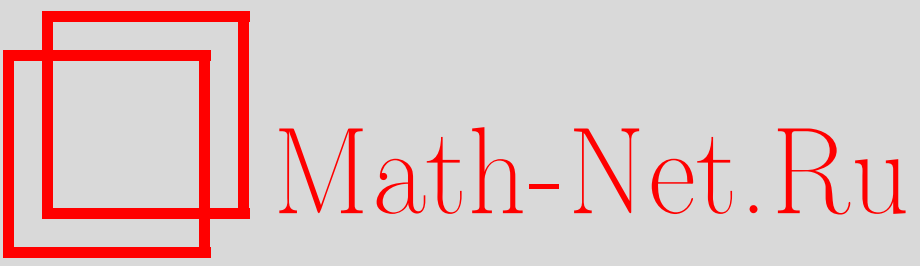

В. С. Владимиров, Я. И. Волович, О нелинейном уравнении динамики в теории p-адической струны, ТМФ, 2004, том 138, номер 3, 355-368

DOI: https://doi.org/10.4213/tmf36

Использование Общероссийского математического портала Math-Net.Ru подразумевает, что вы прочитали и согласны с пользовательским соглашением

http://www . mathnet.ru/rus/agreement

Параметры загрузки:

IP: 54.84 .234 .179

26 апреля 2023 г., 06:06:24 
ТЕОРЕТИЧЕСКАЯ

И МАТЕМАТИЧЕСКАЯ

ФИЗИКА

Том 138, № 3

март, 2004

(C) 2004 r

В.С. Владимиров* ${ }^{*}$ Я. И. Волович ${ }^{\dagger}$

\section{О НЕЛИНЕЙНОМ УРАВНЕНИИ ДИНАМИКИ В ТЕОРИИ $p$-АДИЧЕСКОЙ СТРУНЫ}

Исследуются нелинейные псевдодифференциальные уравнения с бесконечным числом производных. Это уравнения нового класса, первоначально возникшие в теории $p$-адической струны; их исследование представляет интерес для математической физики и ее приложений, в частности в теории струн и в космологии. В настоящей работе предпринято систематическое математическое исследование свойств этих уравнений. Доказана основная теорема единственности решения в некоторой алгебре обобщенных функций, обсуждаются краевые задачи для ограниченных решений и доказана теорема о существовании пространственно-однородных решений при нечетных $p$, а для четных $p$ доказано отсутствие непрерывных неотрицательных решений, интерполирующих между двумя вакуумами, и указывается на возможность наличия разрывных решений. Также рассматривается многомерное уравнение и обсуждаются солитонные и $q$-бранные решения.

Ключевые слова: $p$-адическая струна, псевдодифференциальный оператор, нелинейные уравнения.

\section{1. ВВЕДЕНИЕ}

Недавно в $p$-адической теории струн и обычной теории струн стали исследоваться нелинейные уравнения с бесконечным числом производных [1]-[7] (о p-адической математической физике см. [3], [8]). Исследование этого нового класса уравнений представляет значительный интерес для математической физики, и в настоящей работе предпринято систематическое математическое изучение их свойств.

В струнной теории поля [9] задача построения динамики имеет две существенные особенности по сравнению с аналогичной задачей локальной теории поля. Прежде всего, полевая теория струн соответствует бесконечному набору локальных полей. Во-вторых, получающееся для этих полей взаимодействие нелокально в том смысле, что соответствующие уравнения движения содержат бесконечное число производных.

Интерес к задаче о построении классического решения, интерполирующего между различными вакуумами, связан с возможными применениями в космологии, а именно в

* Математический институт им. В. А. Стеклова РАН, Москва, Россия

${ }^{\dagger}$ Московский государственный университет, Москва, Россия. E-mail: yaroslav@aylabs.com 
работе [5] предложено отождествлять инфлатонное поле с тахионной материей в бозонной струнной теории.

В $p$-адической модели струны для скалярного поля тахионов возникло новое уравнение движения - нелинейное псевдодифференциальное уравнение вида (см. [1], [2], а также обзоры [3], [4] и библиограффию в них)

$$
p^{\square / 2} \Phi=\Phi^{p},
$$

где

$$
\square=\frac{\partial^{2}}{\partial_{t}^{2}}-\frac{\partial^{2}}{\partial_{x_{1}}^{2}}-\ldots-\frac{\partial^{2}}{\partial_{x_{d-1}}^{2}}
$$

- оператор Д'Аламбера и $p$-простое число $(p=2,3, \ldots)$. В настоящей работе мы будем считать $p$ любым целым числом, бо́льшим единицы. Физический интерес представляют только вешественные решения уравнения (1.1), и впредь будут рассматриваться только они. Более общие уравнения и системы получены и изучаются в работах [7], [10].

Напомним, как получается $p$-адическая струна [3]. Хорошо известно, что если в струнной теории рассмотреть рассеяние тахиона, то получается амплитуда Венециано [9], представимая в виде бета-функции над полем вешественных чисел. Если эту бета-функцию заменить соответствуюшей $p$-адической бета-функцией, то получится амплитуда рассеяния тахиона в $p$-адической струне [1], [3]. Регуляризованные адельные формулы для амплитуд Венециано рассмотрены в [11].

Уравнение (1.1) при $p=2$ имеет два вакуумных решения: $\Phi_{0}=0$ и $\Phi_{0}=1$. В недавней работе [6] проведено исследование сушествования решений (1.1), интерполируюших между этими вакуумами. Было показано, что таких монотонных решений не сушествует. В настояшей работе мы докажем более обшую теорему, согласно которой не существует даже немонотонных решений при любом четном $p$. В работах [7], [10] была численно рассмотрена аналогичная задача для тахиона фермионной струны. Здесь будет доказана теорема о сушествовании пространственно-однородных решений уравнения (1.1), интерполируюших между вакуумами $\Phi_{0}=-1$ и $\Phi_{0}=1$ при нечетных $p$.

Структура работы следующая. В разделе 2 описана постановка задачи, в разделе 3 доказывается основная теорема единственности в алгебре обобщенных функций $\widetilde{\mathcal{S}}_{+}^{\prime}$. В четвертом разделе обсуждаются краевые задачи для ограниченных решений уравнения (1.1) и доказывается теорема о сушествовании пространственно-однородных решений при нечетных $p$, а для четных $p$ докывается отсутствие непрерывных неотрицательных решений, интерполируюших между двумя вакуумами, и указывается на возможность наличия разрьвных решений. В разделе 5 рассматривается многомерное уравнение и обсуждаются солитонные и $q$-бранные решения.

\section{2. ПОСТАНОВКА ЗАДАЧИ}

Простейшее уравнение (1.1) при $d=1$ имеет вид

$$
p^{\partial_{t}^{2} / 2} \Phi=\Phi^{p}
$$


Придадим уравнению (2.1) точный смысл. Это уравнение есть формальная запись нелинейного псевдодифференциального уравнения с символом $e^{-\left(\xi^{2} / 2\right) \ln p}$

$$
\frac{1}{2 \pi} \int_{-\infty}^{\infty} \tilde{\Phi}(\xi) \exp \left(-\frac{1}{2} \xi^{2} \ln p-i t \xi\right) d \xi=\Phi^{p}(t),
$$

где $\tilde{\Phi}(\xi)$ - преобразование Фурье (обобшенной) функции $\Phi(t)$ :

$$
\tilde{\Phi}(\xi)=\int_{-\infty}^{\infty} \Phi(t) e^{i \xi t} d t .
$$

Здесь используется теория преобразования фурье обобщенных функций класса $\mathcal{D}^{\prime}$. Их преобразования Фурье являются аналитическими функционалами из пространства $Z^{\prime}[12]$.

Если решение $\Phi(t)$ уравнения (2.2) ищется в классе обобщенных функций медленного роста $\mathcal{S}^{\prime}$, то это уравнение эквивалентно нелинейному интегральному уравнению

$$
\int_{-\infty}^{\infty} \Phi(\tau) H\left[(t-\tau)^{2}\right] d \tau=\Phi^{p}(t)
$$

где ядро $H\left[(t-\tau)^{2}\right]$ равно

$$
\begin{aligned}
H\left(t^{2}\right) & =\frac{1}{2 \pi} \int_{-\infty}^{\infty} \exp \left(-\frac{1}{2} \xi^{2} \ln p-i t \xi\right) d \xi= \\
& =\frac{1}{\sqrt{2 \pi \ln p}} \exp \left(-\frac{t^{2}}{2 \ln p}\right), \quad \int_{-\infty}^{\infty} H\left(t^{2}\right) d t=1 .
\end{aligned}
$$

Уравнение (2.3) эквивалентно (в терминах преобразования Фурье) уравнению в свертKax

где свертка

$$
p^{-\xi^{2} / 2} \tilde{\Phi}(\xi)=\frac{1}{(2 \pi)^{p-1}}(* \tilde{\Phi})^{p}(\xi),
$$

$$
(* \tilde{\Phi})^{p}(\xi)=\underbrace{(\Phi * \Phi * \cdots * \Phi)}_{(p-1) \text { раз }}(\xi) .
$$

Левая часть уравнения (2.3) является значением функционала $\Phi(\tau)$ на основной функции $H\left[(t-\tau)^{2}\right] \in S$, т.е. сверткой

$$
(\Phi * H)(t)=\left(\Phi(\tau), H\left[(t-\tau)^{2}\right]\right) .
$$

Правая часть уравнения (2.3) имеет смысл, если ее рассматривать в алгебре по умножению обобшенных функций $\widetilde{\mathcal{S}}_{+}^{\prime} \subset \mathcal{S}^{\prime}$. Поэтому точный смысл уравнения $(2.3)$ в алгебре $\widetilde{\mathcal{S}_{+}^{\prime}}$ есть

$$
\left(\Phi(\tau), H\left[(t-\tau)^{2}\right]\right)=\Phi^{p}(t) .
$$

Напомним, что $\widetilde{\mathcal{S}}_{+}^{\prime}-$ преобразование Фурье обобщенных функций медленного роста с носителем на полуоси $[0, \infty)$. Алгебра $\widetilde{\mathcal{S}}_{+}^{\prime}$ изоморфна сверточной алгебре граничных значений голоморфных функций $f(z)(z=t+i y)$ в верхней полуплоскости $y>0$, удовлетворяюших условию роста [13]

$$
|f(t+i y)| \leqslant C \frac{1+|z|^{\alpha}}{y^{\beta}}, \quad y>0,
$$

при некоторых $C>0, \alpha \geqslant 0$ и $\beta \geqslant 0$. 


\section{3. ОСНОВНАЯ ТЕОРЕМА ЕДИНСТВЕННОСТИ}

Используя аналитические методы, применяемые в аксиоматической квантовой теории поля, мы докажем здесь следуюшую теорему единственности решения уравнения (2.3) (точнее - уравнения (2.6)).

ТЕОРЕма 1. Если $\Phi(t)$ - вещественное решение уравнения (2.6) из алгебры $\widetilde{\mathcal{S}}_{+}^{\prime}$, mo

$$
\Phi(t)= \begin{cases}0 \text { или } \pm 1, & p \text { - нечетное } \\ 0 \text { или } 1, & p \text {-четное }\end{cases}
$$

ДокАЗАТЕльство. Пусть $\Phi \in \widetilde{\mathcal{S}}_{+}^{\prime}$ есть вешественное решение уравнения (2.6). Тогда $\Phi(t)$ - граничное значение в $\mathcal{S}^{\prime}$ функции $\Phi(z), z=t+i y$, голоморфной в верхней полуплоскости $y>0$ и удовлетворяющей условию роста (2.7).

С другой стороны, из уравнения (2.6) следует, что обобщенная функция $\Phi^{p}(t)$ допускает аналитическое продолжение

$$
\Phi^{p}(z)=C_{1}\left(\Phi(\tau), e^{-\sigma(z-\tau)^{2}}\right), \quad C_{1}=(2 \pi \ln p)^{-1 / 2}, \quad \sigma=(2 \ln p)^{-1},
$$

на всю плоскость комплексной переменной $z$ и удовлетворяет условию роста

$$
\left|\Phi^{p}(z)\right| \leqslant C^{\prime}(1+|z|)^{2 m} e^{\sigma y^{2}}, \quad z \in \mathbb{C},
$$

где $m$ - порядок обобшенной функции $\Phi$ и $C^{\prime}>0$.

Докажем это утверждение. Тот факт, что правая часть равенства (3.2) есть целая функция, а ее граничное значение при $y \rightarrow 0$ равно правой части уравнения (2.6) и, стало быть, совпадает с функцией $\Phi^{p}(t)$, устанавливается стандартными методами [13].

Докажем оценку (3.3). Из равнства (3.2) выводим

$$
\begin{aligned}
\left|\Phi^{p}(z)\right| & \leqslant C_{1}\|\Phi\|_{-m}\left\|e^{-\sigma(z-\tau)^{2}}\right\|_{m} \leqslant C_{2} \max _{0 \leqslant j \leqslant m} \sup _{\tau}\left(1+|\tau|^{j}\right)\left|\frac{d^{j}}{d \tau^{j}} e^{-\sigma(z-\tau)^{2}}\right| \leqslant \\
& \leqslant C_{3} \sup _{\tau}\left(1+|\tau|^{m}\right)\left(1+|z-\tau|^{m}\right)\left|e^{-\sigma\left(z^{2}-2 z \tau+\tau^{2}\right)}\right| \leqslant \\
& \leqslant C_{3}\left(1+|z|^{m}\right) e^{\sigma\left(y^{2}-t^{2}\right)} \sup _{\tau}\left(1+|\tau|^{m}\right) e^{-\sigma\left(\tau^{2}-2 t \tau\right)}
\end{aligned}
$$

Докажем, что

$$
\sup _{\tau}\left(1+|\tau|^{m}\right) e^{-\left(\tau^{2}-2 t \tau\right)} \leqslant C_{4}\left(1+|t|^{m}\right) e^{\sigma t^{2}} .
$$

При $|t| \leqslant 1$ оценка (3.5) очевидна. Докажем ее для $|t|>1$. Обозначим

$$
f(\rho,|t|)=\rho^{m} e^{-\sigma\left(\rho^{2}-2 \rho|t|\right)}, \quad \rho \geqslant 0
$$

Тогда

$$
\sup _{\tau}|\tau|^{m} e^{-\sigma\left(\tau^{2}-2 \tau|t|\right)} \leqslant \sup _{\tau} f(\tau,|t|)=f\left(\rho_{0},|t|\right), \quad|t|>1
$$


Точка максимума $\rho_{0}$ функции $f(\rho,|t|)$ удовлетворяет уравнению

$$
\rho^{2}-|t| \rho-\frac{m}{2 \sigma}=0
$$

откуда

$$
|t| \leqslant \rho_{0}=\frac{|t|}{2}+\sqrt{\frac{t^{2}}{4}+\frac{m}{2 \sigma}} \leqslant|t|+\frac{m}{\sigma|t|}, \quad|t|>1 .
$$

Подставляя это значение $\rho_{0}$ в оценку (3.6), получим

$$
\sup _{\tau}|\tau|^{m} e^{-\sigma\left(\tau^{2}-2 \tau|t|\right)} \leqslant C_{5}\left(1+|t|^{m}\right) e^{\sigma t^{2}}, \quad|t|>1
$$

Отсюда следует справедливость (3.5) при всех $t$. Из (3.4), (3.5) следует оценка (3.3), откуда получаем

$$
|\Phi(z)| \leqslant C_{6}(1+|z|)^{2 m / p} e^{\sigma y^{2} / p}, \quad z \in \mathbb{C} .
$$

Теперь докажем оценку

$$
|\Phi(z)| \leqslant C_{7}(1+|z|)^{n}, \quad z \in \mathbb{C},
$$

при некоторых $C_{7}>0$ и $n \geqslant 0$.

Введем функцию

$$
\Phi_{1}(z)= \begin{cases}\Phi(z), & y>0 \\ \bar{\Phi}(\bar{z}), & y<0 .\end{cases}
$$

Поскольку граничные значения $\Phi_{1}(x \pm i 0)=\Phi(t)$ функции $\Phi_{1}(z)$ совпадают (обобщенная функция $\Phi(t)$ вещественна), то по теореме Боголюбова об “острие клина" [13] функция $\Phi_{1}(z)$ целая и, стало быть, $\Phi_{1}(z)=\Phi(z), z \in \mathbb{C}$. В силу $(2.7) \Phi_{1}(z)$ удовлетворяет оценке

$$
\left|\Phi_{1}(z)\right| \leqslant C \frac{1+|z|^{\alpha}}{|y|^{\beta}}, \quad z \in \mathbb{C},
$$

но она же удовлетворяет и (3.8). Поэтому

$$
|\Phi(z)| \leqslant \min \left\{C \frac{1+|z|^{\alpha}}{|y|^{\beta}}, C_{6}(1+|z|)^{2 m / p} e^{\sigma y^{2} / p}\right\}
$$

откуда следует оценка (3.9).

По теореме Лиувилля из (3.10) следует, что целая функция $\Phi(z)$ есть полином степени не выше $n$ :

$$
\Phi(z)=\sum_{k=0}^{n} a_{k} z^{k}
$$

и поэтому

$$
\tilde{\Phi}(\xi)=\sum_{k=0}^{n} 2 \pi(-i)^{k} a_{k} \delta^{(k)}(\xi) .
$$


Подставляя выражение (3.12) для $\tilde{\Phi}(\xi)$ в уравнение (2.5), получим

$$
p^{-\xi^{2} / 2} \sum_{k=0}^{n}(-i)^{k} a_{k} \delta^{(k)}(\xi)=\left(* \sum_{k=0}^{n}(-i)^{k} a_{k} \delta^{(k)}\right)^{p}(\xi) .
$$

Поскольку система обобщенных функций $\left\{\delta^{(k)}, k=0,1, \ldots\right\}$ линейно независима, то из (3.13) и того, что $p n>n$ при $n>0$, выводим

$$
(-1)^{n p} a_{n}^{p} \delta^{(p n)}(\xi)=0 .
$$

Поэтому $a_{n}=0$, и т.д. В результате получаем, что в представлениях (3.11) и (3.12) $a_{k}=$ $0, k=1,2, \ldots, n$. Поэтому формулы $(3.11)$ и (3.12) принимают вид $\tilde{\Phi}(\xi)=2 \pi a_{0} \delta(\xi)$ и $\Phi(t)=a_{0}$. Из уравнения $(2.5)$ легко видеть, что возможные значения постоянной $a_{0}$ равны 0 и 1 при четном $p$ и 0 и \pm 1 при нечетном $p$. Теорема 1 доказана.

\section{4. КРАЕВЫЕ ЗАДАЧИ ДЛЯ ОГРАНИЧЕННЫХ РЕШЕНИЙ}

Уравнение (2.3) имеет быстро растушее решение

$$
\Phi(t)=\exp \left(\frac{\ln p}{2(p-1)}+\frac{p-1}{2 p \ln p} t^{2}\right),
$$

что проверяется непосредственно с использованием формулы

$$
e^{-\alpha t^{2}} * e^{\beta t^{2}}=\sqrt{\frac{\pi}{\alpha-\beta}} \exp \left(\frac{\alpha \beta}{\alpha-\beta} t^{2}\right), \quad \alpha>\beta .
$$

Расмотрим ограниченные решения $\Phi(t)$ уравнения (2.3). Возникает вопрос: какими дополнительными свойствами они будут обладать? Ведь функция $\Phi^{p}(t)$ есть след при $y=0$ целой функции

$$
F(z)=\int_{-\infty}^{\infty} H\left[(z-\tau)^{2}\right] \Phi(\tau) d \tau, \quad z=t+i y \in \mathbb{C}
$$

(см. доказательство теоремы 1 ), т.е. $\Phi(t)$ удовлетворяет алгебраическому уравнению

$$
\Phi^{p}(t)=F(t), \quad t \in \mathbb{R},
$$

где $F(t)$ - ограниченная вещественно-аналитическая функция. Здесь нужно различать два случая: $p$ - нечетное и $p$ - четное.

При нечетном $p$ ограниченное (вешественное) решение уравнения (4.1) единственно и равно $F^{1 / p}(t)$. Оно вешественно-аналитическое там, где $F(t) \neq 0$.

При четном $p$ не исключены решения с разрывами первого рода. Например, в точке $t_{0}$, где $F\left(t_{0}\right)>0$, решением может служить функция вида

$$
\Phi(t)= \begin{cases}F^{1 / p}(t), & t>t_{0}, \\ -F^{1 / p}(t), & t<t_{0} .\end{cases}
$$


О НЕЛИНЕЙНОМ УРАВНЕНИИ ДИНАМИКИ В ТЕОРИИ $p$-АДИЧЕСКОЙ СТРУНЫ 361

Чтобы избежать экзотических решений уравнения (4.1), таких, например, как

$$
\Phi(t)= \begin{cases}F^{1 / p}(t), & \text { если } t-\text { иррациональное } \\ -F^{1 / p}(t), & \text { если } t-\text { рациональное }\end{cases}
$$

сузим класс рассматриваемых решений уравнения (2.3) (при четном $p$ ) до класса ограниченных кусочно-вещественно-аналитических функций. Построенные разрывные решения (4.2) уравнения (4.1) - кусочно-вешественно-аналитические.

Однако остается открытым вопрос о нахождении таких точек $t_{0}$. Возможно, ими являются вещественные нули производной целой функции $F^{\prime}(z)$, т.е. такие точки $t_{0}$, что $F^{\prime}\left(t_{0}\right)=0$.

Теперь рассмотрим решения уравнения (4.1) в окрестности тех точек, где $F\left(t_{0}\right)=0$. Это нуль целой функции $F(z)$, и потому найдутся целое число $n>0$ и вешественноаналитическая функция $F_{1}(t) \neq 0$ (при четном $p \quad F_{1}(t)>0$ и $n$ - четно) такие, что в окрестности точки $t_{0}$

$$
F(t)=\left(t-t_{0}\right)^{n} F_{1}(t)
$$

Поэтому все возможные решения при четном $p$

$$
\Phi(t)=\left\{\begin{array}{l} 
\pm\left(t-t_{0}\right)^{n / p} F_{1}^{1 / p}(t), \quad t>t_{0}, \\
\pm\left(t_{0}-t\right)^{n / p} F_{1}^{1 / p}(t), \quad t<t_{0},
\end{array}\right.
$$

и одно решение при нечетном $p$

$$
\Phi(t)=\left(t-t_{0}\right)^{n / p} F_{1}^{1 / p}(t)
$$

уравнения (4.1) непрерывны в точке $t_{0}$ и являются кусочно-вешественно-аналитическими в окрестности $t_{0}$

Резюмируя, приходим к следуюшему выводу.

При нечетном р всякое ограниченное решение уравнения (2.3) является непрерывным и кусочно-вещественно-аналитическим; при четном р всякое ограниченное кусочно-непрерывное решение - кусочно-вещественно-аналитическое, при этом возмохний скачок решения $\Phi(t)$ в точках разрыва $t_{0}$ равен либо $2 \Phi\left(t_{0}\right)$, либо $-2 \Phi\left(t_{0}\right)$.

Возникают следуюшие вопросы. Сушествуют ли вешественно-аналитические решения уравнения (2.3)? Сушествуют ли при четном $p$ разрывные решения уравнения (2.3)? Ответ на второй вопрос могут дать численные методы.

ТЕОРема 2. Если решение (не обязательно вещественное) $\Phi(t)$ уравнения $(2.3)$ ограничено, то оно удовлетворяет оченке

$$
|\Phi(t)| \leqslant 1, \quad t \in \mathbb{R}
$$


ДокАЗАТЕЛЬСтво. По предполжению решение $\Phi(t)$ ограничено. Поэтому сушествует такое число $M>0$, что

$$
\sup _{t}|\Phi(t)|=M .
$$

Из уравнений (2.3) и (2.2) следует цепочка

$$
\begin{aligned}
\left|\Phi^{p}(t)\right| & =|\Phi(t)|^{p}=\left|\int_{-\infty}^{\infty} \Phi(t) H\left[(t-\tau)^{2}\right] d \tau\right| \leqslant \int_{-\infty}^{\infty}|\Phi(t)| H\left[(t-\tau)^{2}\right] d \tau \leqslant \\
& \leqslant \sup _{t}|\Phi(t)| \int_{-\infty}^{\infty} H\left[(t-\tau)^{2}\right] d \tau=M,
\end{aligned}
$$

откуда получаем неравенство

$$
\sup _{t}|\Phi(t)|^{p}=\left[\sup _{t}|\Phi(t)|\right]^{p}=M^{p} \leqslant M,
$$

так что $M \leqslant 1$. Теорема 2 доказана.

Другие свойства ограниченных непрерывных решений уравнения (2.3) можно найти в работе [6]. В частности там доказано, что не сушествует монотонно возрастаюших решений $\Phi(t)$ уравнения (2.3), удовлетворяюших граничным условиям

$$
\lim \Phi(t)=\left\{\begin{array}{l}
0, t \rightarrow-\infty \\
1, t \rightarrow+\infty
\end{array}\right.
$$

Докажем, что ограниченных решений, удовлетворяюших условиям (4.5), вообше не сушествует. Справедливо даже более сильное утверждение.

Теорема 3. Не существует неотрицательных ограниченных непрерывных решений $\Phi(t)$ краевой задачи

$$
\lim \Phi(t)=1, \quad t \rightarrow+\infty
$$

для уравнения (2.3) и для некоторого $t_{0}$ такого, что $\Phi\left(t_{0}\right) \leqslant 2^{-1 /(p-1)}$.

ДокАЗАТЕЛЬСтво. По теореме 2 имеем $0<\Phi(t)<1$. Из условий $(4.6)$ докажем, что сушествует $t_{1}>t_{0}$ такое, что $\Phi\left(t_{1}\right)<\Phi\left(t_{0}\right)$. Пусть такого $t_{1}$ нет. Тогда при всех $t \geqslant t_{0}$ было бы справедливо неравенство $\Phi(t) \geqslant \Phi\left(t_{0}\right)$. Но при этом из уравнения $(2.3)$ следовало бы

$$
\Phi^{p}\left(t_{0}\right)=\int_{-\infty}^{\infty} \Phi(\tau) H\left[\left(t_{0}-\tau\right)^{2}\right] d \tau>\Phi\left(t_{0}\right) \int_{t_{0}}^{\infty} H\left[\left(t_{0}-\tau\right)^{2}\right] d \tau=\frac{\Phi\left(t_{0}\right)}{2},
$$

что противоречит неравенству (4.6).

Обозначим через $M$ множество всех $t>t_{0}$ таких, что $\Phi(t)<\Phi\left(t_{0}\right)$. Исходя из доказанного, множество $M$ не пусто. Обозначим $\sup _{t \in M} t=T$. Это значит, что существует возрастаюшая последовательность $\left\{t_{k}, k=0,1, \ldots\right\}$ точек множества $M$ такая, что $t_{k} \rightarrow T$. Если $T<\infty$, то вследствие непрерывности функции $\Phi(t) \Phi\left(t_{k}\right) \rightarrow \Phi(T)$, 
О НЕЛИНЕЙНОМ УРАВНЕНИИ ДИНАМИКИ В ТЕОРИИ $p$-АДИЧЕСКОЙ СТРУНЫ 363

$k \rightarrow \infty$. Но значение $\Phi(T) \leqslant \Phi\left(t_{0}\right)$ удовлетворяет неравенству (4.6). Поэтому сушествует точка $T_{1}>T$ такая, что $\Phi\left(T_{1}\right)<\Phi(T) \leqslant \Phi\left(t_{0}\right)$, а это противоречит определению точки $T$. Итак, $T=\infty$. Но тогда

$$
\Phi\left(t_{k}\right)<\Phi\left(t_{0}\right) \leqslant 2^{-1 /(p-1)}<1,
$$

что противоречит предельному соотношению (4.6). Теорема 3 доказана.

Из теоремы 3 , как следствие, выводим: не существует неотрицательных ограниченных непрерывных решений краевой задачи (2.3), (4.5).

Теорема 4. Существует единственное неотрицательное ограниченное непрерьвное решение $\Phi(t) \equiv 1$ краевой задачи

$$
\lim \Phi(t)= \begin{cases}1, & t \rightarrow-\infty \\ 1, & t \rightarrow+\infty\end{cases}
$$

для уравнения $(2.3)$.

ДокАЗАТЕЛЬСТво. Действительно, $\Phi(t) \equiv 1$ есть решение краевой задачи $(2.3)$, (4.7). Пусть существует другое решение этой задачи $0 \leqslant \Phi(t) \not \equiv 1$. Из доказанного соотношения $0<\Phi(t)<1$ и в силу (4.7) сушествует $t_{0}$ такое, что

$$
0<\Phi\left(t_{0}\right)=\min _{t} \Phi(t)<1 .
$$

Но тогда из уравнения $(2.3)$

$$
\Phi^{p}\left(t_{0}\right)=\int_{-\infty}^{\infty} H\left[\left(t_{0}-\tau\right)^{2}\right] \Phi(\tau) d \tau \geqslant \Phi\left(t_{0}\right),
$$

откуда следует $\Phi\left(t_{0}\right) \geqslant 1$, что противоречит (4.8). Полученное противоречие доказывает теорему 4 .

Вопрос о существовании и единственности других ограниченных решений уравнения (2.3), кроме указанных выше, остается открытым. Тем не менее численные вычисления показывают, что для начальной функции

$$
\Phi_{0}(t)=\operatorname{sgn} t
$$

при $p=3$ итерации

$$
\Phi_{n}(t)=\left(\int_{-\infty}^{\infty} \Phi_{n-1}(\tau) H\left[(t-\tau)^{2}\right] d \tau\right)^{1 / p}, \quad n=1,2, \ldots,
$$

быстро и равномерно сходятся [10].

Здесь мы без использования численных методов докажем, что эти итерации при любом нечетном $p$ равномерно сходятся к решению краевой задачи

$$
\lim \Phi(t)= \begin{cases}-1, & t \rightarrow-\infty, \\ 1, & t \rightarrow+\infty,\end{cases}
$$

для уравнения $(2.3)$. 
ЛЕмма. Если $\Phi(t)$ - ограниченная функиия на $\mathbb{R} u$

$$
\lim _{t \rightarrow+\infty} \Phi(t)=1
$$

mo

$$
\lim _{t \rightarrow+\infty} \int_{-\infty}^{\infty} H\left[(t-\tau)^{2}\right] \Phi(\tau) d \tau=1 .
$$

ДоКАЗАТЕЛЬСтво. В силу (2.4) и (4.12) получаем равенство (4.13):

$$
\begin{aligned}
\lim _{t \rightarrow+\infty} & \int_{-\infty}^{\infty} H\left[(t-\tau)^{2}\right] \Phi(\tau) d \tau= \\
& =\lim _{t \rightarrow+\infty} \int_{0}^{\infty} H\left[(t-\tau)^{2}\right] \Phi(\tau) d \tau+\lim _{t \rightarrow+\infty} \int_{-\infty}^{0} H\left[(t-\tau)^{2}\right] \Phi(\tau) d \tau= \\
& =\lim _{t \rightarrow+\infty} \int_{-t}^{\infty} H\left[u^{2}\right] \Phi(t+u) d u+\lim _{t \rightarrow+\infty} \int_{t}^{\infty} H\left[u^{2}\right] \Phi(t-u) d u= \\
& =\int_{-\infty}^{\infty} H\left[u^{2}\right] \lim _{t \rightarrow+\infty} \Phi(t+u) d u+O\left(\lim _{t \rightarrow+\infty} \int_{t}^{\infty} H\left[u^{2}\right] d u\right)= \\
& =\int_{-\infty}^{\infty} H\left[u^{2}\right] d u=1 .
\end{aligned}
$$

В первом интеграле мы воспользовались теоремой Лебега, а во втором - ограниченностью функции $\Phi(t)$. Лемма доказана.

Теорема 5. Пусть $p$ - нечетное. Тогда существует нечетное непрерывное решение краевой задачи (2.3), (4.11).

ДоКАЗАТЕЛЬСтво. Если ограниченное решение сушествует, то согласно доказанному выше оно непрерывно. Поскольку нас интересуют нечетные решения, то задача $(2.3),(4.11)$ эквивалентна следующей задаче для функции $\varphi(t)=\Phi(t \sqrt{2 \ln p})$ :

$$
\int_{0}^{\infty} \mathcal{K}(t, \tau) \varphi(\tau) d \tau=\varphi^{p}(t), \quad t \geqslant 0
$$

и

$$
\lim _{t \rightarrow \infty} \varphi(t)=1 .
$$

Здесь

$$
\mathcal{K}(t, \tau)=\frac{1}{\sqrt{\pi}}\left[e^{-(t-\tau)^{2}}-e^{-(t+\tau)^{2}}\right]
$$

- симметричное непрерьвное положительное ядро, обрашаюшееся в нуль при $t=0$ или $\tau=0$. При этом

$$
\Phi(t)=\left\{\begin{array}{cc}
\varphi\left(\frac{t}{\sqrt{2 \ln p}}\right), \quad t \geqslant 0, \\
-\varphi\left(\frac{t}{\sqrt{2 \ln p}}\right), \quad t<0 .
\end{array}\right.
$$


Воспользуемся итерационным процессом (4.10), который в терминах соответствующих функций $\varphi_{n}(t)$ будет иметь вид

$$
\varphi_{n}^{p}(t)=\int_{0}^{\infty} \mathcal{K}(t, \tau) \varphi_{n-1}(\tau) d \tau, \quad \varphi_{0}(t)=1, \quad t \geqslant 0, \quad n=0,1, \ldots
$$

Значение первой итерации $\varphi_{1}(t)$ задается в виде

$$
\varphi_{1}(t)=\operatorname{erf}(t)^{1 / p}
$$

где функция ошибок $\operatorname{erf}(t)$ определяется как

$$
\operatorname{erf}(t)=\frac{2}{\sqrt{\pi}} \int_{0}^{t} \exp \left(-x^{2}\right) d x
$$

Из (4.19) и (4.20) следует, что

$$
0 \leqslant \varphi_{1}(t)<1=\varphi_{0}(t)
$$

для всех $t \geqslant 0$.

Докажем равномерную ограниченность и сходимость итерационного процесса (4.18).

Заметим, что из $\varphi_{0}(t)=1$ и (4.17) согласно лемме следует, что для всех номеров итераций $n \geqslant 0$ выполняется

$$
\lim _{t \rightarrow \infty} \varphi_{n}(t)=1 .
$$

Докажем теперь, что для первой и второй итерации процесса (4.18) имеет место неравенство

$$
\sigma \varphi_{1}(t) \leqslant \varphi_{2}(t) \leqslant \varphi_{1}(t), \quad t \geqslant 0,
$$

при некотором $\sigma$, причем

$$
0<\sigma<1
$$

Докажем сначала вторую часть неравенства (4.23). Для этого заметим, что

$$
\varphi_{2}(t)^{p}=\int_{0}^{\infty} \mathcal{K}(t, \tau) \varphi_{1}(\tau) d \tau \leqslant \int_{0}^{\infty} \mathcal{K}(t, \tau) d \tau=\operatorname{erf}(t)=\varphi_{1}(t)^{p}
$$

где переход к неравенству возможен ввиду (4.21).

При $t=0$ неравенство (4.23) обрашается в равенство и таким образом выполняется. Рассмотрим теперь случай строго положительных $t$.

Определим функцию $f(t)=\varphi_{2}(t)^{p} / \varphi_{1}(t)^{p}$. Она непрерывна, положительна и, согласно (4.25), $f(t) \leqslant 1$.

Воспользовавшись правилом Лопиталя, вычислим предел в точке $t=0$. Для этого заметим, что

$$
\frac{d}{d t} \varphi_{1}^{p}(t)=\frac{d}{d t} \operatorname{erf}(t)=\frac{2}{\sqrt{\pi}} e^{-t^{2}},\left.\quad \frac{d}{d t} \varphi_{1}^{p}(t)\right|_{t=0}=\frac{2}{\sqrt{\pi}}
$$




$$
\begin{aligned}
\frac{d}{d t} \varphi_{2}^{p}(t) & =\int_{0}^{\infty} \frac{d}{d t} \mathcal{K}(t, \tau) \varphi_{1}(\tau) d \tau= \\
& =\frac{2}{\sqrt{\pi}} \int_{0}^{\infty}\left[-(t-\tau) e^{(t-\tau)^{2}}+(t+\tau) e^{(t+\tau)^{2}}\right] \operatorname{erf}(\tau)^{1 / p} d \tau
\end{aligned}
$$

откуда

$$
\left.\frac{d}{d t} \varphi_{2}^{p}(t)\right|_{t=0}=\frac{4}{\sqrt{\pi}} \int_{0}^{\infty} e^{-\tau^{2}} \tau \operatorname{erf}(\tau)^{1 / p} d \tau
$$

Тогда из (4.26) и (4.28) следует

$$
\lim _{t \rightarrow 0} f(t)=2 \int_{0}^{\infty} e^{-\tau^{2}} \tau \operatorname{erf}(\tau)^{1 / p} d \tau<2 \int_{0}^{\infty} e^{-\tau^{2}} \tau d \tau=1 .
$$

С другой стороны, согласно (4.22)

$$
\lim _{t \rightarrow \infty} f(t)=\lim _{t \rightarrow \infty} \frac{\varphi_{2}(t)^{p}}{\varphi_{1}(t)^{p}}=1 .
$$

Предел (4.29) позволяет рассматривать функцию $f(t)$ как непрерывную на $[0, \infty)$, тогда в силу непрерьвности с учетом (4.30) сушествует $\delta>0: f(t) \geqslant \delta>0$. Используемая в (4.23) величина $\sigma$ определяется как $\sigma=\delta^{1 / p}$. Таким образом, выполнение неравенства (4.23) доказано.

Воспользовавшись свойством положительности ядра $\mathcal{K}(t, \tau)$, мы можем проинтегрировать неравенство (4.23):

$$
\sigma \int_{0}^{\infty} \mathcal{K}(t, \tau) \varphi_{1}(\tau) d \tau \leqslant \int_{0}^{\infty} \mathcal{K}(t, \tau) \varphi_{2}(\tau) d \tau \leqslant \int_{0}^{\infty} \mathcal{K}(t, \tau) \varphi_{1}(\tau) d \tau
$$

что приводит к

$$
\sigma \varphi_{2}(t)^{p} \leqslant \varphi_{3}(t)^{p} \leqslant \varphi_{2}(t)^{p}
$$

или

$$
\sigma^{1 / p} \varphi_{2}(t) \leqslant \varphi_{3}(t) \leqslant \varphi_{2}(t)
$$

и т.д. В итоге получаем

$$
\sigma^{1 / p^{n-1}} \varphi_{n}(t) \leqslant \varphi_{n+1}(t) \leqslant \varphi_{n}(t)
$$

откуда

$$
0 \leqslant \varphi_{n}(t)-\varphi_{n+1}(t) \leqslant \varphi_{n}(t)\left(1-\sigma^{1 / p^{n-1}}\right) .
$$

Ввиду того, что из $(4.21)$ и $(4.33)$ следует $0 \leqslant \varphi_{n}(t)<1$, имеем

$$
\left|\varphi_{n}(t)-\varphi_{n+1}(t)\right|<\frac{-\ln \sigma}{p^{n-1}}, \quad n \geqslant 1 .
$$

Здесь мы воспользовались неравенством

$$
1-\sigma^{\alpha}<-\alpha \ln \sigma
$$


О НЕЛИНЕЙНОМ УРАВНЕНИИ ДИНАМИКИ В ТЕОРИИ $p$-АДИЧЕСКОЙ СТРУНЫ 367

которое выполняется при любом $\alpha>0$ и $\sigma \in(0,1)$. Для доказательства (4.36) рассмотрим функцию

$$
f(\sigma)=-\alpha \ln \sigma+\sigma^{\alpha}-1 .
$$

Мы видим, что $f(0) \rightarrow+\infty, f(1)=0$ и ее производная отришательна:

$$
f^{\prime}(\sigma)=-\frac{\alpha}{\sigma}+\alpha \sigma^{\alpha-1}=\frac{\alpha}{\sigma}\left(\sigma^{\alpha}-1\right)<0 .
$$

Заметим, что в силу (4.24) правая часть неравенства (4.35) всегда положительна. Из (4.35) следует равномерная сходимость последовательности $\varphi_{n}(t)$ при $t \leqslant 0$.

Докажем теперь, что функция $\varphi(t)$, определяемая как

$$
\varphi(t)=\lim _{n \rightarrow \infty} \varphi_{n}(t)=\varphi_{0}(t)+\sum_{n=0}^{\infty}\left[\varphi_{n+1}(t)-\varphi_{n}(t)\right],
$$

удовлетворяет граничному условию (4.15).

Как доказано выше, ряд (4.37) сходится равномерно по $0 \leqslant t<\infty$, поэтому, используя (4.22) и переходя к пределу при $t \rightarrow \infty$, получим

$$
\lim _{t \rightarrow \infty} \varphi(t)=\lim _{t \rightarrow \infty} \varphi_{0}(t)+\lim _{t \rightarrow \infty} \sum_{n=0}^{\infty}\left[\varphi_{n+1}(t)-\varphi_{n}(t)\right]=1+\sum_{n=0}^{\infty}[1-1]=1,
$$

что и доказывает свойство (4.15), а с ним и исходную теорему.

Очевидно также, что отраженная функция $\Phi(-t)$ есть также решение уравнения (2.3), удовлетворяющее отраженным граничным условиям (4.11) $\lim _{t \rightarrow \pm \infty} \Phi(t)=\mp 1$. Вопрос о единственности такого типа решений по-прежнему остается открытым.

\section{5. МНОГОМЕРНЫЕ УРАВНЕНИЯ ДВИЖЕНИЯ $p$-АДИЧЕСКОЙ СТРУНЫ, $d>1$}

Повторяя рассуждения, приведенные в разделе 2, убеждаемся, что уравнение (1.1) есть $d$-мерное псевдодифференциальное уравнение вида

$$
\frac{1}{(2 \pi)^{d-1}} \int_{\mathbb{R}^{d}} \tilde{\Phi}_{x}(\tau, \xi) H\left[(t-\tau)^{2}\right] \exp \left(\frac{1}{2}|\xi|^{2} \ln p-i(x, \xi)\right) d \tau d \xi=\Phi^{p}(t, x),
$$

где $\tilde{\Phi}_{x}(\tau, \xi)$ - преобразование Фурье по переменным $x=\left(x_{1}, \ldots, x_{d-1}\right)$,

$$
|\xi|^{2}=\xi_{1}^{2}+\cdots+\xi_{d-1}^{2}, \quad(x, \xi)=x_{1} \xi_{1}+\cdots+x_{d-1} \xi_{d-1} .
$$

Соответствуюшее одномерное уравнение по переменной $x_{j}=x$ имеет вид (cp. (2.1))

$$
\Psi^{p}(x)=\frac{1}{2 \pi} \int_{-\infty}^{\infty} \tilde{\Psi}(\xi) \exp \left(\frac{1}{2} \xi^{2} \ln p-i x \xi\right) d \xi
$$


Оно имеет солитонное решение (ср. (4.1))

$$
\Psi(x)=\exp \left(\frac{\ln p}{2(p-1)}-\frac{p-1}{2 p \ln p} x^{2}\right)
$$

и тривиальные решения, указанные в теореме 1 раздела 2.

С помошью солитонных решений (5.3) строятся $q$-бранные $(q=0,1, \ldots, d-2)$ солитонные решения уравнения (5.1) (см. работу [14] и библиографию в ней)

$$
\Phi\left(x_{q+1}, \ldots, x_{d-1}\right)=\Psi\left(x_{q+1}\right) \Psi\left(x_{q+2}\right) \ldots \Psi\left(x_{d-1}\right),
$$

не зависяшие от $t, x_{1}, \ldots, x_{q}$.

Физический смысл солитонных решений (5.4) обсуждается во многих работах, в частности в [4]-[6], [10], [14].

Благодарности. Авторы выражают глубокую благодарность И. Я. Ареф̆ьевой и И. В. Воловичу за полезные обсуждения. Работа выполнена при финансовой поддержке гранта президента РФ по поддержке ведуших научных школ НШ-1542.2003.1, а также грантов РФФИ № 02-01-01084 и МАС-03-01-06466.

\section{Список литературы}

[1] L. Brekke, P. G. Freund, M. Olson, E. Witten. Nucl. Phys. B. 1988. V. 302. P. 365.

[2] P. H. Frampton, Y. Okada. Phys. Rev. D. 1988. V. 37. № 10. P. 3077.

[3] В. С. Владимиров, И. В. Волович, Е. И. Зеленов. Р-адический анализ и математическая физика. М.: Наука, 1994.

[4] L. Brekke, P. G. O. Freund. Phys. Rep. 1993. V. 233. № 1. P. 1.

[5] A. Sen. JHEP. 2002. V. 204. P. 48; hep-th/0203211.

[6] N. Moeller, B. Zwiebach. JHEP. 2002. V. 210. P. 34; hep-th $/ 0207107$.

[7] I. Ya. Aref'eva, L. V. Joukovskaya, A. S. Koshelev. JHEP. 2003. V. 309. P. 12; hep-th/0301137.

[8] A. Yu. Khrennikov. p-Adic Valued Distributions in Mathematical Physics. Dordrecht: Kluwer Acad. Publ., 1994.

[9] М. Грин, Дж. Швари, Э. Виттен. Теория суперструн. М.: Мир, 1990.

[10] Ya. Volovich. J. Phys. A. 2003. V. 36. P. 8685; math-ph/0301028.

[11] V. S. Vladimirov. Lett. Math. Phys. 1993. V. 28. P. 123.

[12] И. М. Гельфанд, Г. Е. Шилов. Обобщенные функции. Вып. 2. Пространства основных и обобщенных функций. М.: Физматлит, 1958.

[13] В. С. Владимиров. Методы теории функций многих комплексных переменных. М.: Наука, 1964.

[14] D. Ghoshal, A. Sen. Nucl. Phys. B. 2000. V. 584. P. 300.

Поступила в редакцию 6.III.2003 г., после доработки 28.IV.2003 г. 